\title{
Von der Verwundbarkeit der Menschen und Gottes
}

Überlegungen im Anschluss an Dietrich Bonhoeffer

Dominik Weyl

"Vulnerability, on the other hand, is

a perfection of loving freedom. $\aleph^{1}$

William C. Placher

\section{Von der Verwundbarkeit der Menschen}

Es würde nicht recht Sinn machen, an den Beginn meines Beitrags Zahlen und Statistiken zu stellen. Zum Beispiel, wie viele kriegsversehrte Menschen sich noch immer aus dem Süden zu uns auf die Flucht begeben und wohl ihre Heimat, nicht jedoch ihre Traumata hinter sich lassen. Oder wie hoch der statistische Anteil derjenigen in der Schweizerischen Bevölkerung ist, die an Körper und Seele Leid tragen und sich darum gegenwärtig in ärztlicher Betreuung befinden. Oder wie wahrscheinlich es ist, dass unsere gesellschaftlichen Strukturen und Verhaltensweisen dazu beitragen, dass nicht nur die schwächsten Glieder der Gesellschaft, sondern auch die vermeintlich Starken gekränkt, verletzt, getroffen werden. Es braucht keine Beweise für die vielen unterschiedlichen und unterschiedlich schweren Verwundungen - es reicht unser Wissen von der Welt und unsere eigene Erfahrung: Wir sind verwundbar.

Seit den 1980er Jahren hat der Begriff /Verwundbarkeit` oder , Vulnerabilität in einigen akademischen Fächern besonderes Interesse gefunden: Zuerst in der Armutsforschung, später dann in der Medizin und der Psychologie, in der philosophischen Ethik und Anthropologie, in der Konflikt- und der Friedensforschung, aber auch in der Hydrogeologie und Agrarökonomik. Die Theologie scheint dieses Interesse bisher noch nicht recht zu teilen. Dabei könnten, so ist Hildegund Keul überzeugt, aus den in diesen Fächern bereits

1 William C. Placher, Narratives of a Vulnerable God. Christ, Theology, and Scripture, Louisville, KY 1994, 19. 
geführten Debatten bedeutsame Einsichten auch für die Theologie gezogen werden. Und andersherum könnte die Theologie relevante Perspektiven in diese Debatten einbringen, da ihr Themen wie Verwundung, Leid, Armut und Gewalt keineswegs fremd seien. Keul hält vor allem die im Vulnerabilitätsdiskurs gemachte Feststellung theologisch für bedenkenswert, dass menschliches Leben - bezogen sowohl auf seine individuelle Verfasstheit als auch hinsichtlich seiner gesellschaftlichen Beziehungen und Strukturen - verwundbar, vulnerabel sei. ${ }^{2}$ Mit der Philosophin Judith Butler teilt Keul darum die »Feststellung, dass die körperliche und soziale Verletzlichkeit das menschliche Leben unausweichlich konstituiert ${ }^{3}$.

In der Theologie ist individuelle wie soziale Verletzlichkeit immer wieder durchdacht worden. Ihr Wissen von der Welt und ihre eigene Erfahrung hat Theologinnen und Theologen fragen lassen ,Warum leiden wir?. Diese Frage hat in der sog. Theodizeefrage eine spezifische Zuspitzung gefunden, auf die es durch die Jahrhunderte hindurch zahlreiche Antwortversuche gegeben hat. Je für sich genommen konnten diese Versuche die Theodizeefrage zwar nicht beantworten, aber in ihrer Kontingenz haben sie sich doch auch nicht zu Georg Büchners »Fels des Atheismus « ${ }^{4}$ verdichtet.

Nicht von der Hand zu weisen ist wohl Keuls Hinweis, dass die Theologie bisher eher zu einer "Fokussierung auf zugefügte Wunden ${ }^{5}$ neigte, statt Verwundbarkeit zu thematisieren. Einen solchen Ansatz vertreten seit gut fünf Jahrzehnten etwa die politische und öffentliche Theologie, indem sie besonderes Augenmerk auf die sozialen Wunden legen, die sich Menschen in persönlichen Beziehungen, durch gesellschaftliche Strukturen und weltweite, ökonomische und ökologische Zusammenhänge zufügen. Exemplarisch dafür steht noch immer Dorothee Sölles Buch Leiden (1973). Sölle fragt darin nicht "nach der Notwendigkeit und Möglichkeit der Abschaffung des Elends, sondern [...] nach den Subjekten dieses Prozesses. Wer arbeitet an der Aufhebung gesellschaftlicher Zustände,

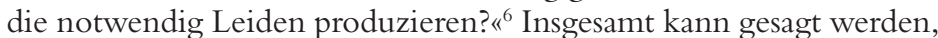
dass die Theologie auch durch Theologinnen wie Sölle gelernt hat, dass es ihr hinsichtlich der unterschiedlichen Verwundungen heute

\footnotetext{
2 Vgl. für diesen Absatz Hildegund Keul, Verwundbarkeit - eine unerhörte Macht. Christliche Perspektiven im Vulnerabilitätsdiskurs, in: Herder Korrespondenz 69 (2015), 647-651, hier 647f.

3 Ebd., 648.

4 Georg Büchner, Dantons Tod. Ein Drama, Stuttgart 2005, 3. Akt, 1. Szene, 52.

5 Keul,Verwundbarkeit - eine unerhörte Macht, 648.

${ }^{6}$ Dorothee Sölle, Leiden, Stuttgart ${ }^{6} 1984,9$ [Hervorhebung durch den Verfasser, künftig: HdV].
} 
und Verwundbarkeit im Allgemeinen nicht (mehr) um eine "Sinngebung des Sinnlosen" gehen kann, weil die Theologie auch von dort her »an die Grenzen ihrer Systematisierungsfähigkeit geführt [wird] und [...] dies auch artikulieren [muss] «. ${ }^{7}$ Jedoch ist es eine Aufgabe der Theologie, die seelsorgerliche und diakonische Begleitung der an Leib und Seele Versehrten durch kirchliche Institutionen kritisch zu reflektieren und (Korrektur-)Vorschläge für die Praxis zu machen. Auch ist es ihre Aufgabe, Wege zur Vermeidung neuer Verwundungen zu suchen, indem sie nach den Bedingungen und Strukturen fragt, unter denen Verletzung, Kränkung und Beleidigung heute möglich sind. Dabei wird sich die Theologie auch in die öffentliche Debatte einschalten müssen, um auf diese Bedingungen und Strukturen einzuwirken und somit Heilung und Aussöhnung zu fördern. Die Fürsorge der Theologie für die Verwundeten und Verwundbaren birgt in sich schon jene "Zukunftskategorie», die Keul durch den Begriff , Vulnerabilität s stark machen will - ihr geht es weniger um erlittene Verwundungen, sondern um Verwundbarkeit als eine anthropologische Konstituente sowie um die der Verwundbarkeit "entgegenwirkenden Schutzstrategien ${ }^{8}{ }^{8}$

Auch wenn Verletzlichkeit das menschliche Leben "unausweichlich konstituiert ${ }^{9}$, bleibt die Grundkonstituente des Menschen aus theologischer Sicht »das eigentümliche Verhältnis von Mensch und Gott" (DBW $3^{10}, 58$ ) - so Dietrich Bonhoeffer in seiner Vorlesung Schöpfung und Fall (1932/33). In dieser Vorlesung, einer systematischen Auslegung von Gen 1-3, beschreibt Bonhoeffer die Ebenbildlichkeit des Menschen als "die von Gott selbst gesetzte Beziehung" (DBW 3, 61) zu ihm. Die biblischen Schöpfungsberichte bringen die Bezogenheit Gottes auf den Menschen zum Ausdruck - eine für Bonhoeffers Theologie grundlegende Einsicht. Dass Gott sein Ebenbild nicht nur schafft, sondern auch segnet, bedeute weiterhin, dass Gott den Menschen »bejaht [...] ganz in der Welt des Lebendigen, in die er gestellt ist, es ist seine ganz empirische Existenz, die hier gesegnet wird«(DBW 3, $64[\mathrm{HdV}])$. Das ist die Bedingung, unter

\footnotetext{
7 Oswald Bayer, Art. Leiden. 2. Dogmatisch und ethisch, in: ${ }^{4}$ RGG, Bd. 5, Tübingen 2002, 243-246, hier 244f.

${ }^{8}$ Keul, Verwundbarkeit- eine unerhörte Macht, 648. Beachtenswert ist, dass Keul ausdrücklich darauf hinweist, dass solche Schutzstrategien auch dazu führen können, dass Menschen und Menschengruppen, die verletzenden Strukturen und Gewalt ausgesetzt sind, für sich zugleich solche Strukturen schaffen, denen zu ihrem Schutz selbst ein Gewaltpotential gegen andere eignet (vgl. ebd., 647f.).

9 Ebd., 648 .

${ }^{10}$ Dietrich Bonhoeffer, Schöpfung und Fall, Dietrich Bonhoeffer Werke (DBW), Bd. 3, hg.v. Martin Rüter/Ilse Tödt, München 1989.
} 
der der Mensch existiert. ${ }^{11}$ Seine Geschöpflichkeit und der Segen Gottes umfassen demnach alle Dimensionen und Bedingtheiten des Menschen: seine Weltbezüge, seine sozialen Beziehungen, seinen Leib und seine Seele. Und darin eben auch seine Verwundbarkeit. So sind die Menschen, verbunden mit Gott, ihren Mitmenschen und der Welt, ganz Mensch.

Der zweite Schöpfungsbericht des Alten Testaments endet mit derVertreibung des Menschen aus dem Paradies, dem Garten Eden (Gen 3,24). In mythologischer Sprache wird dort beschrieben, dass der Mensch mit seinen Mitmenschen fortan in einer Welt steht und wirkt, in der er angreifbar ist und in der er selbst angreift; in der er verwundbar ist und in der er selbst verwundet.

"Da kam Jesus mit ihnen zu einem Garten, der hieß Gethsemane» (Mt 26,36). Im Gegensatz zum Paradiesgarten ist - beispielsweise für Sölle - Gethsemane zum symbolischen Ort für die Verwundbarkeit der Menschen geworden. Die Evangelien berichten: Judas hatte Jesus verraten und die Knechte der Hohepriester und Ältesten waren schon auf dem Weg hinauf zum Ölberg, um ihn dort gefangen zu nehmen. Das Passionsgeschehen ist ausgelöst. Jesus »fing an zu trauern und zu zagen", er sonderte sich von den Jüngern ab, "fiel nieder auf sein Angesicht und betete und sprach: Mein Vater, ist's möglich, so gehe dieser Kelch an mir vorüber" (Mt 26,37.39). Das LukasEvangelium weicht an dieser Stelle von den beiden anderen synoptischen Evangelien ab: Auf das Gebet Jesu hin »erschien ihm aber ein Engel vom Himmel und stärkte ihn « (Lk 22,43). ${ }^{12}$ Bei Matthäus und Markus bleibt Jesus in seiner Verwundbarkeit alleine. Kein Engel kommt, ihn zu stärken. Sölle schreibt dazu: "Gott schweigt, wie so oft in der Geschichte des Menschen, Jesus bleibt allein mit seinem wiederholten Schrei, seiner Todesangst, seiner wahnsinnigen Hoffnung, seinem bedrohten Leben. « $^{13}$ Diese Lesart der Passionsgeschichten war - auch abseits ihrer sozialpolitischen Ausrichtung - vor 50 Jahren durchaus anstössig. Erst in dieser Zeit begannen Theologinnen und Theologen ausdrücklich Verrat, Gefangennahme, Spott, Folter und Kreuz Jesu als das zu verstehen, was sie waren: echtes Leid, echte Verwundung, echter Tod. Dass Jesus trauert und zagt, dass er Angst und Agonie erleidet, wurde nun starken traditionellen Ansätzen

${ }_{11}$ Vgl. Dietrich Bonhoeffer, Berlin 1932-1933, DBW, Bd. 12, hg.v. Carsten Nicolaisen/Ernst-Albert Scharffenorth, Gütersloh 1997, $183 f$.

${ }^{12} \mathrm{Da}$ es sich bei Lk 22,43 wohl um eine frühe Einfügung in den griechischen Text handelt, stützt dieser Vers die nachfolgenden Beobachtungen, dass bereits das antike Christentum daran interessiert war, Jesus als möglichst leidensunfähig und heroisch auszuweisen. (Vgl. den Kommentar zu diesem Vers in ${ }^{27} \mathrm{NA}$ ).

${ }^{13}$ Sölle, Leiden, $100 f$. 
entgegengehalten, die, beeinflusst durch die griechische Philosophie, über die Jahrhunderte versuchten, »aus Jesus ein heroisches Wesen $\mathrm{zu}$ machen $\aleph^{14}$, das apathisch seine Verwundbarkeit erdulde, ja gar nicht leiden könne (sog. Apathieaxiom). Christiane Tietz summiert: "Die Hauptlinie der christlichen Theologie hat bis ins 20. Jahrhundert hinein an diesem Dogma von der Leidensunfähigkeit Gottes festgehalten. ${ }^{15}$ Bonhoeffer hat diese Ansätze seinerzeit bereits kritisiert. In Gethsemane zeige sich gerade »die Umkehrung von allem, was der religiöse Mensch von Gott erwartet «:»Leiden Gottes in Jesus Christus«. (DBW 8 ${ }^{16}, 535 f$.) Neben der Leidensunfähigkeit Gottes wurde ferner auch der exklusive Charakter derVerwundbarkeit Jesu bestritten. Jesus habe nicht anders gelitten als die Menschen vor und nach ihm, auch habe er ihnen gegenüber nicht "am meisten" gelitten, sondern so wie ihm "kann es allen ergehen $"{ }^{17}$ Wir sind verwundbar. "Da sprach Jesus zu ihnen: Meine Seele ist betrübt bis an den Tod" (Mt 26,38). Nach Sölle liegt "die Wahrheit des Symbols [...] gerade in seiner Wiederholbarkeit « ${ }^{18}$. Die Erfahrung Jesu in Gethsemane wird so paradigmatisch als die allgemein-menschliche Erfahrung individueller wie sozialer Verletzlichkeit gedeutet.

\section{Von der Verwundbarkeit Gottes}

Die Verwundbarkeit Jesu weist den theologischen Blick weiter zum Kreuz. Auf Golgatha soll sie nun nicht mehr nur für seine Jünger, sondern für alle sichtbar werden: Und als sie ihn verspottet hatten, zogen sie ihm den Mantel aus und zogen ihm seine Kleider an und führten ihn ab, um ihn zu kreuzigen" (Mt 27,31). Keul würdigt, dass die Theologie der letzten Jahrzehnte am Kreuz »dieVerpflichtung zu Compassion und Solidarität « abgelesen und eine "Sensibilität für die Opfer der Anderen" entwickelt habe, die sie fruchtbar in den Vulnerabilitätsdiskurs einbringen könne. ${ }^{19}$ Dazu müsse sie aber auch bereit sein, die »Fokussierung auf zugefügte Wunden« zu überschreiten, die

\footnotetext{
${ }^{14}$ Ebd., 102.

${ }^{15}$ Christiane Tietz, Der leidende Gott, in: Panorama. Intercultural Annual of Interdisciplinary Ethical and Religious Studies for Responsible Research 23 (2011), 107-120, hier 110.

${ }^{16}$ Dietrich Bonhoeffer, Widerstand und Ergebung. Briefe und Aufzeichnungen aus der Haft, DBW, Bd. 8, hg.v. Christian Gremmels/Eberhard Bethge/Renate Bethge, Gütersloh 1998.

${ }^{17}$ Sölle, Leiden, 104.

18 Ebd.

${ }^{19}$ Keul,Verwundbarkeit - eine unerhörte Macht, 648.
} 
Keul darauf zurückführt, dass das Leiden Jesu am Kreuz als "Leidensparadigma" für die allgemein-menschliche Erfahrung von Verletzlichkeit zu stark ins Zentrum gerückt worden sei. ${ }^{20}$ Hinsichtlich des interdisziplinären Diskurses benötige es jedoch "nicht nur eine Thematisierung von Wunden, sondern auch von Vulnerabilität « ${ }^{21}$.

Tatsächlich steht eine Fokussierung auf das Leiden Jesu theologisch in der Gefahr, das Leiden Gottes in Jesus Christus zu übersehen. Das hat bereits Martin Luther bemerkt: "Denn wenn ich das gleube, das allein die menschliche natur fur mich gelidden hat, so ist mir der Christus ein schlechter heiland, so bedarff er wol selbs eines heilands $\aleph^{22}$. Wenn aber nur ein Schritt hinter die Ansätze zurückgegangen wird, die Keul im Blick hat, kann gezeigt werden, dass die Theologie zu der von ihr eingeforderten Thematisierung von Vulnerabilität durchaus fähig ist, indem sie gerade Kreuzestheologie bleibt. Anhand einer Interpretation von Bonhoeffers Rede vom sleidenden Gott soll dies nun ausgeführt werden.

In der deutschsprachigen Theologie war Bonhoeffer der erste, der vom `leidenden Gott gesprochen hat. ${ }^{23}$ Überlegungen dazu klingen bereits in seiner Christologie-Vorlesung (1933) an, vollends zur Sprache kommen sie aber erst etwa zehn Jahre später in seinen Briefen und Aufzeichnungen aus dem Gefängnis, die nach Bonhoeffers Tod unter dem Titel Widerstand und Ergebung veröffentlicht wurden. Zwar hat er während seiner Aufenthalte in New York (1930/31) und London (1935-37) in der zeitgenössischen anglo-amerikanischen Theologie wahrscheinlich vergleichbare Ansätze ${ }^{24}$ kennengelernt, aber es war Bonhoeffer, der durch seine Überlegungen »das Ende der (vorschnellen) Selbstverständlichkeit Gottes« eingeläutet und "so eine Art Trendwende in der christlichen Gotteslehre" eingeleitet hat. ${ }^{25}$

In der Christologie-Vorlesung kommt Bonhoeffers theologische Nähe zu Luther deutlich zum Ausdruck. Der Reformator hat zeitlebens energisch an der communicatio idiomatum festgehalten, die wiederum auf einen Beschluss des Konzils von Chalcedon (451)

\footnotetext{
${ }^{20}$ Ebd., 648.

${ }^{21}$ Ebd.

${ }^{22}$ Martin Luther,Vom Abendmahl Christi, Bekenntnis (1528), WA 26, 319, 37-39.

${ }^{23}$ Vgl. Jürgen Moltmann, Theologie mit Dietrich Bonhoeffer. Die Gefängnisbriefe, in: John W. de Gruchy/Stephen Plant/Christiane Tietz (Hg.), Dietrich Bonhoeffers Theologie heute. Ein Weg zwischen Fundamentalismus und Säkularismus?, Gütersloh 2009, 17-31, hier 29.

${ }^{24}$ Vgl. zu diesen Ansätzen Tietz, Der leidende Gott, 115-118.

25 Jan Bauke-Ruegg, Die Allmacht Gottes. Systematisch-theologische Erwägungen zwischen Metaphysik, Postmoderne und Poesie, Berlin/New York 1998, 36.
} 
zurückgeht: Jesus Christus sei als "wahrhaft Gott und wahrhaft Mensch ${ }^{26}$ zu bekennen. Luther schreibt: "[...] weil Gottheit vnd menscheit ynn Christo eine Person ist / so gibt die schrifft / vmb solcher personlicher einickeit willen auch der Gottheit / alles was der menschheit widderferet / vnd widderümb ${ }^{27}$. In Jesus ist Gott ganz Mensch geworden. Diese theologische Einsicht ist auch für Bonhoeffer zentral, "daß Gott ganz ist, wo er ist" (DBW 12, 335). Und nur auf der Grundlage dieser Einsicht, dass Gott immer ganz da ist, wo er ist, kann von Gott und kann vom Menschen in rechter Weise gesprochen werden. Darum fährt Bonhoeffer später fort: "[Weder] von Gott noch vom Menschen kann etwas gewußt werden, bevor Gott Mensch geworden ist in Jesus Christus." (DBW 12, 339) Für Bonhoeffer muss daher jeder Versuch, abseits von Gottes Offenbarung in Jesus Christus über Gott und über den Menschen $\mathrm{zu}$ sprechen, in Idealismus und Abstraktion abgleiten. ${ }^{28}$ Michael Mawson fasst deshalb treffend zusammen: „For Bonhoeffer, theology should simply attend to God's revelation in Christ; it should not seek to speculate or speak about God (or humanity) more directly than this. ${ }^{29}$ Sein theologisches Denken und insbesondere seine Überlegungen zum >leidenden Gott < beginnt Bonhoeffer darum mit »der Gegebenheit, Jesus Christus ist Gott" (DBW 12, 336). Diese Gegebenheit beschreibt nicht die Vergottung Jesu von Nazareth, sondern im Gegenteil die Menschwerdung Gottes in diesem Menschen. Sie beantwortet mithin die Frage, "Wer ist dieser Gott? Er ist der Menschgewordene wie wir Menschen geworden sind. Er ist ganz Mensch."(DBW 12, 340)

Weil Gott in Jesus Christus ganz Mensch geworden ist, ist ihm nichts Menschliches fremd, keine Verletzungen und keine Narben, nicht das Leid und nicht die Not der Menschen. Gott lässt sich in Jesus Christus ganz auf ein Menschsein ein, das verwundbar ist. ${ }^{30}$ Bonhoeffer distanziert sich darum schon in der Christologie-Vorlesung von der bis dahin weitgehend selbstverständlichen Vorstellung eines

\footnotetext{
${ }^{26}$ Konzil von Chalkedon, in: Heinrich Denzinger, Kompendium der Glaubensbekenntnisse und kirchlichen Lehrentscheidungen, hg.v. Peter Hünermann, Freiburg i.Br. u.a. ${ }^{37} 1991,140-143$, hier 142.

${ }^{27}$ Luther,Vom Abendmahl Christi, Bekenntnis, WA 26, 321, $5-7$.

${ }^{28}$ Vgl. Michael Mawson, How God Suffers. Bonhoeffer, Moltmann and Theological Language, Vortrag auf dem 6. International Bonhoeffer Colloquium: Dietrich Bonhoeffer's Concept of Theology, Zürich, 30.10.-01.11.2014 (im Erscheinen).

${ }^{29}$ Ebd.

${ }^{30}$ Vgl. Christiane Tietz, Krankheit und die Verborgenheit Gottes, in: Günter Thomas/ Isolde Karle (Hg.), Krankheitsdeutung in der postsäkularen Gesellschaft. Theologische Ansätze im interdisziplinären Gespräch, Stuttgart 2009, 354-366, hier 364.
} 
allmächtigen göttlichen Wesens: "Soll Jesus Christus als Gott beschrieben werden, so darf nicht von seiner Allmacht und Allwissenheit geredet werden, sondern von seiner Krippe und seinem Kreuz." (DBW 12, 341) In der Krippe und am Kreuz Jesu spiegelt sich die Verwundbarkeit Gottes wider: Armut, Bedrängnis und Flucht, Verfolgung, Verwundung, Schmerz und Tod erfährt auch Gott. Die Krippe, aber vor allem das Kreuz werden zu den Orten, »an [denen] Gott sein Wesen zeigt und von [denen] her Gottes Gottsein zu verstehen ist $^{31}$, wie mit Tietz festgehalten werden kann. Erfährt Jesus Verwundungen und Leid, so ist Gott selbst verwundet und leidet. "Denn wenn ich das gleube, das allein die menschliche natur fur mich gelidden hat, $[\ldots]$ so bedarff er wol selbs eines heilands $\aleph^{32}$, hatte Luther geschrieben.

In seinem Entwurf für eine Arbeit fragt Bonhoeffer im August 1944 erneut: "Wer ist Gott?» und skizziert eine Antwort: "Nicht zuerst ein allgemeiner Gottesglaube an Gottes Allmacht etc." (DBW 8, 558). In den Wochen und Monaten zuvor hat sich Bonhoeffer mit den tradierten biblischen Begriffen und religiösen Vorstellungen angesichts der unzweifelhaften Mündigkeit der Menschen und der Welt auseinandergesetz $\mathrm{t}^{33}-$ da sass er schon mehr als ein Jahr im Wehrmachtsuntersuchungsgefängnis Berlin-Tegel ein. Wer ist Gott? "Begegnung mit Jesus Christus." (DBW 8, 558)

Im Matthäus-Evangelium wird berichtet: "[...] und er trieb die Geister aus durch sein Wort und machte alle Kranken gesund, auf dass erfüllt würde, was gesagt ist durch den Propheten Jesaja, der da spricht: 'Er hat unsre Schwachheit auf sich genommen, und unsre Krankheiten hat er getragen'" (Mt 8,16b-17 mit Jes 53,4). Bezugnehmend auf dieseVerse verdeutlicht Bonhoeffer, dass der christliche Gott nicht allmächtig abseits der Welt über die Welt und die Men-

\footnotetext{
${ }^{31}$ Tietz, Der leidende Gott, hier 111; mit Bezug auf Eberhard Jüngel,Vom Tod des lebendigen Gottes. Ein Plakat, in: ZThK 65 (1968), 93-116, hier 108.

${ }^{32}$ Luther,Vom Abendmahl Christi, Bekenntnis, WA 26, 319, 37-39.

${ }_{33}$ Bonhoeffer diagnostiziert: "Gott als moralische, politische, naturwissenschaftliche Arbeitshypothese ist abgeschafft, überwunden; ebenso aber als philosophische und religiöse Arbeitshypothese (Feuerbach!). Es gehört zur intellektuellen Redlichkeit, diese Arbeitshypothese fallen zu lassen bzw. sie so weitgehend wie irgend möglich auzuschalten. [...] Wo behält nun Gott noch Raum? fragen ängstliche Gemüter, und weil sie darauf keine Antwort wissen, verdammen sie die ganze Entwicklung, die sie in solche Notlage gebracht hat. [...] Und wir können nicht redlich sein, ohne zu erkennen, daß wir in der Welt leben müssen - setsi deus non daretur [ [auch wenn es keinen Gott gäbe ‘]. Und eben dies erkennen wir - vor Gott! [...] So führt uns unser Mündigwerden zu einer wahrhaftigen Erkenntnis unsrer Lage vor Gott." (DBW 8, 532f.)
} 
schen herrscht, sondern - sichtbar vor allem am Kreuz - »kraft seiner Schwachheit, seines Leidens" (DBW 8, 534). Das widerspricht allen selbstverständlichen Erwartungen an einen Gott. Der Erwartung beispielsweise, dass Gott apathisch und leidensunfähig sich nicht bewegen liesse von menschlicher Verwundbarkeit. Der Erwartung auch, dass sich erst durch die Differenz Gottes zur Welt seine Eigenschaften ergeben würden. Oder schliesslich der Erwartung, dass Gott dann handelt, wenn die Menschen die Grenzen ihrer Handlungsfähigkeit erreicht haben - oder nur meinen, an diese Grenzen gestossen zu sein. ${ }^{34}$ "Was ein Gott, so wie wir ihn uns denken, alles tun müßte und könnte, damit hat der Gott Jesu Christi nichts zu tun." (DBW 8, 572) Darin unterscheiden sich - Bonhoeffer unternimmt in der Haft vor allem eine theologische Religionskritik ${ }^{35}$ - die Religiösen von den Christen:

"Die Religiösen sprechen von Gott, wenn menschliche Erkenntnis (manchmal schon aus Denkfaulheit) zu Ende ist oder wenn menschliche Kräfte versagen - es ist eigentlich immer der deus ex machina, den sie aufmarschieren lassen, entweder zur Scheinlösung unlösbarer Probleme oder als Kraft bei menschlichem Versagen, immer also in Ausnutzung menschlicher Schwäche bzw. an den menschlichen Grenzen; [... - ich möchte von Gott nicht an den Grenzen, sondern in der Mitte [...] sprechen.» (DBW 8, 407f.)

Nicht also mit einem metaphysischen Gott, sondern mit dem Gott, der in Jesus Christus ganz Mensch geworden ist, haben es die Menschen zu tun. Nicht mit einem, der zwar ihren natürlichen Erwartungen an einen Gott entspricht, darin aber gerade ein unzuverlässiger Gott ist: Ein vom Theaterdonner umstürmter deus ex machina, der sehr wohl frei, aber in seiner Freiheit willkürlich handelt. Mit dieser "falschen Gottesvorstellung" (DBW 8, 535) beginnt Bonhoeffer im Gefängnis aufzuräumen. Seine früheren Überlegungen aus der Christologie-Vorlesung aufgreifend, legt er nun dar, dass Gott in der Welt nicht durch seine Allmacht wirkt, sondern dass Gott "durch seine Ohnmacht in der Welt Macht und Raum gewinnt" (DBW 8, 535). Bonhoeffer beschreibt Gott darum zunächst passiv als den Gott, der leidet, weil er - ganz Mensch - in Jesus Christus Verfolgung, Schmerz und Tod erleidet. Seit seiner Gefangennahme in

\footnotetext{
${ }^{34}$ Vgl. Tietz, Der leidende Gott, 112.

${ }^{35}$ Vgl. dazu Christiane Tietz, Unzeitgemäße Aktualität. Religionskritik in Zeiten der "Wiederkehr der Religion", in: Ingolf U. Dalferth/Hans-Peter Grosshans (Hg.), Kritik der Religion. Zur Aktualität einer unerledigten Aufgabe, Tübingen 2006, 243-258.
} 
Gethsemane liegt Jesu Geschick in den Händen anderer. Das ist die Umkehrung der bis dahin selbstverständlichen Gottesvorstellung, zu der Bonhoeffer in der Haft gelangt ist.

Er ist überzeugt, dass es die Menschen mit diesem Gott zu tun haben: Dieser Gott »läßt sich aus der Welt herausdrängen ans Kreuz« (DBW 8, 534 [HdV]) und zeigt sich in der Welt verwundbar. Darüber kommt Bonhoeffer zur Formulierung eines Satzes, der sicherlich zu seinen bekanntesten zählt: "Die Bibel weist den Menschen an die Ohnmacht und das Leiden Gottes; nur der leidende Gott kann helfen" (DBW 8, 534). Gerade in der Passivität seines Leidens, seiner Verwundbarkeit, ist Gott höchst aktiv und hilft. Er hilft dabei nicht durch sein Mitleid, denn Mitleid heilt keine Wunden. Gott hilft, weil er in Jesus Christus mit leidet: "Christus [...] erfuhr alles Leiden aller Menschen an seinem Leibe als eigenes Leiden - ein unbegreiflich hoher Gedanke! -, er nahm es auf sich in Freiheit." (DBW 8, 34) Und im Moment der schlimmsten Verwundung, dem Tod Jesu am Kreuz, erleidet Gott ram eigenen Leib das ärgste Leid, das Menschen - freilich aus theologischer Perspektive - widerfahren kann: das Gefühl der Gottverlassenheit. Weil er nicht eingreift, weil er unsere Verwundung nicht wunderbar heilt, muss sich Gott von uns abgewandt haben. »Und zu der neunten Stunde rief Jesus laut: [...] Mein Gott, mein Gott, warum hast du mich verlassen?« (Mk $15,34)$ Aber es ist - paradox genug - Jesus, der dort ruft. Die Verwundbarkeit der Menschen und das Leid, das sie erfahren, weil sie verwundbar sind, werden am Kreuz »neu qualifiziert « ${ }^{36}$. Das heisst: Verwundungen werden nicht einfach geheilt, Vernarbungen werden immer sichtbar sein - der Mensch bleibt verwundbar. Aber das Verhältnis von menschlicher Verwundbarkeit und Gott hat sich verändert: Gott steht an der Seite derVerwundbaren und Verwundeten. ${ }^{37}$ Im Dezember 1943 schreibt Bonhoeffer in einem Weihnachtsbrief an seine Eltern:

"Daß Elend, Leid, Armut, Einsamkeit, Hilflosigkeit und Schuld vor den Augen Gottes etwas ganz anderes bedeuten als im Urteil der Menschen, daß Gott sich gerade dorthin wendet, wo die Menschen sich abzuwenden pflegen, daß Christus im Stall geboren wurde, weil er sonst keinen Raum in der Herberge fand, - das begreift ein Gefangener besser als ein anderer und das ist für ihn wirklich eine frohe Botschaft $[\ldots]$.. (DBW 8,241)

\footnotetext{
36 Tietz, Der leidende Gott, 113.

${ }^{37}$ Vgl. ebd., mit Bezug auf Jürgen Moltmann, Die Theodizeefrage und der Schmerz Gottes, in: ders., In der Geschichte des dreieinigen Gottes. Beiträge zur trinitarischen Theologie, unver. Nachdruck der 1. Aufl. 1991, Gütersloh [2010], 54-58, hier 57 f.
} 
Krippe und Kreuz - dort gewinnt Gott Macht und von dort her hilft Gott. Für Bonhoeffer ist die Rede vom `leidenden Gott die Konkretion seiner grundlegenden Einsicht von der Bezogenheit Gottes auf die Menschen. ${ }^{38}$ Gott wird in Jesus Christus ganz Mensch für mich. Und für mich macht sich Gott verwundbar und erleidet Armut, Bedrängnis und Flucht,Verfolgung,Verwundung, Schmerz und Tod. Diesen Aspekt betonte Bonhoeffer bereits in seiner ChristologieVorlesung: "Das Sein der Person Christi ist wesenhaft Bezogenheit auf mich." (DBW 12, 295) Die Konkretion von Krippe und Kreuz wehrt jede Spekulation über Gottes Sein in Jesus Christus ab, jede Abstraktion und jeden Idealismus, denn in der Krippe und am Kreuz zeigt Gott sein Wesen. «D.h. daß ich Jesus Christus nie in seinem An-Sich-Sein denken kann, sondern nur in seiner Bezogenheit auf mich." (DBW 12, 295f.)

\section{Von der Wirksamkeit der freien Liebe Gottes}

Ohnmächtig gewinnt Gott Macht. Bonhoeffer streicht nicht - wie spätere Theologinnen und Theologen - die Allmacht Gottes aus dem theologischen Vokabular. Im Entwurf für eine Arbeit schreibt er: "Wer ist Gott? Nicht zuerst ein allgemeiner Gottesglaube an Gottes Allmacht etc. [...] Aus der Freiheit von sich selbst, aus dem >Für-andere-dasein < bis zum Tod entspringt erst die Allmacht, Allwissenheit, Allgegenwart." (DBW 8, 558 [HdV]) Gott wählt die Ohnmacht um der Menschen willen; er wählt sie aus freier Liebe. Worin die Macht dieser freien Liebe besteht, hat Tietz präzisiert:

"Die >Macht< dieser Liebe besteht darin, dass sie das Leid des andern nicht zurückweichen lässt. Denn es gibt nichts, was die Liebe fürchten müsste aus Angst, daran zugrunde zu gehen. Während menschlicher Liebe das Leid des anderen zu viel werden kann, gilt dies für Gottes Liebe nicht. Gottes Liebe - genau das ist ihre Allmacht - vermag alles zu ertragen, ohne daran zu zerbrechen. « $^{39}$

(All-)Macht wird bei Bonhoeffer zu einem Begriff, der Gottes Bezogenheit auf die Menschen und seine Nähe zu ihnen zum Ausdruck bringt. Gottes Allmacht ist keine Übermacht. Sie ergibt sich vielmehr aus seiner freien Liebe. Aufgrund dieser Liebe entscheidet sich Gott, dieVerwundungen der Menschen mit zu erleiden. Gerade weil

${ }^{38} \mathrm{Vgl}$. Mawson, How God suffers.

39 Tietz, Der leidende Gott, 119. 
Gott liebt, macht er sich verwundbar am Kreuz. Bei Luther heisst es:»Und das ist die Liebe des Kreuzes, [...] die sich dorthin wendet, nicht, wo sie Gutes findet, [...] sondern wo sie dem Schlechten und Bedürftigen Gutes bringen kann. ${ }^{40}$

Etwa im Gegensatz zu Sölle, die - durchaus im Anschluss an Bonhoeffer ${ }^{41}$ - am Kreuz tatsächlich so etwas wie ein $>$ Leidensparadigmar abgelesen hat, vertritt Bonhoeffer mit seiner Rede vom >leidenden Gott< also ein anderes theologisches Interesse. Gott leidet in Jesus Christus nicht zuerst wie wir, sondern mit uns und für uns. Darum ist es auch nicht zuerst das Leiden Gottes, das in seinen Überlegungen zentral scheint. Das ist ja die theologische Herausforderung, vom Leiden Gottes an sich nicht schon in etwas erhöhtem Ton zu reden - ein Eindruck, der sich bei Sölle gelegentlich nahelegt. Dagegen hat William C. Placher in den 1990er Jahren vorgeschlagen, nicht das Leiden Gottes, sondern Gottes Vulnerabilität zum theologischen Bezugspunkt zu machen. Er ist überzeugt, dass sich nicht das Leiden Gottes an sich, sondern Gottes freie Liebe zu seiner freien Schöpfung am Kreuz mitteile. Durch diese freie Liebe, die ihrerseits den Menschen und der Welt Freiheit und Mündigkeit ermöglicht und zugesteht, mache sich Gott verwundbar. ${ }^{42}$ Ein Gedanke, den Placher unter Bezugnahme auch auf Bonhoeffer formuliert. Denn anders gesagt: Aufgrund dieser freien Liebe >lässt sich Gott aus der Welt herausdrängen ans Kreuzı. Placher schliesst daraus: „God suffers because God is vulnerable, and God is vulnerable because God loves - and it is love, not suffering or even vulnerability, that is finally the point. «3 Damit ist Bonhoeffers eigentliches Anliegen seiner Rede vom `leidenden Gott präzise getroffen. Er schreibt: "Christus entzog sich solange dem Leiden, bis seine Stunde gekommen war; dann aber ging er ihm in Freiheit entgegen, ergriff es und überwand es." (DBW 8, 34)

Gottes Bezogenheit auf die Menschen hört dort - und vielleicht gerade dort - nicht auf, wo sich mancher Unversehrte und manche Gesunde bisweilen gerne abwenden würde. Dort zum Beispiel, wo kriegsversehrte Menschen sich auf die Flucht begeben und ihre

\footnotetext{
${ }^{40}$ Martin Luther, Heidelberger Disputation (1518), LDStA 1, 61, 23-26; lateinisches Original:»Et iste amor crucis [...], qui illuc sese transfert, non ubi invenit bonum [...], sed ubi bonum conferat malo et egeno." (Ebd., 60, 19-21).

${ }^{41}$ Vgl. H. Martin Rumscheidt, Dorothee Soelle. Variations on Themes by Dietrich Bonhoeffer, in: Matthew D. Kirkpatrick (Hg.), Engaging Bonhoeffer.The Impact and Influence of Bonhoeffer's Life and Thought, Minneapolis, MN 2016, 169-186, hier 179f. u. 182.

${ }^{42}$ Vgl. Placher, Narratives of a Vulnerable God, 19.

${ }^{43}$ Ebd., 18.
} 
Heimat, nicht jedoch ihre Traumata hinter sich lassen. Oder dort, wo die an Körper und Seele Leid Tragenden gegenwärtig auf ärztliche Betreuung angewiesen sind. Oder dort, wo durch gesellschaftliche Strukturen und Verhaltensweisen nicht nur die schwächsten Glieder der Gesellschaft, sondern auch die vermeintlich Starken gekränkt, verletzt, getroffen werden. Kurz: »Gottes Liebe ist überall wirksam, auch dort, wo Menschen kein ungebrochenes, gelungenes, gesundes Leben vorzuweisen haben. ${ }^{44}$ Das ist das Anliegen der Rede vom >leidenden Gott: Sie will kein >Leidensparadigma vorstellen, sondern sie will zur Sprache bringen, dass Gott nicht abseits der verwundbaren Menschen steht. Gott macht die Menschen nicht unverwundbar, er nimmt nicht einfach ihre Verletzungen und ihre Narben fort. Aber Gott lässt sich in Jesus Christus auf ihre Verwundbarkeit ganz ein, lässt sich davon bewegen und macht sich darum selbst verwundbar. Gottes Verwundbarkeit ist die Vollendung seiner Liebe in Freiheit. ${ }^{45}$ Denn das ist ja die Liebe des Kreuzes, die sich dorthin wendet, wo sie den Verwundeten und den Verwundbaren Gutes bringen kann. Und wenn auch kein Engel kommt, ihn zu stärken: Jesus bleibt nicht allein in Gethsemane - und auch nicht die Menschen in ihrem von Verwundbarkeit bedrohten Leben. ${ }^{46}$

- Dominik Weyl ist Wissenschaftlicher Assistent bei Prof. Dr. Christiane Tietz am Lehrstuhl für Systematische Theologie in Zürich. Er arbeitet an einer Dissertation zur Theologie Dietrich Bonhoeffers.

${ }_{44}$ Tietz, Krankheit und Verborgenheit Gottes, $365 f$.

${ }^{45}$ Vgl. Placher, Narratives of a Vulnerable God, 19.

46 Verbunden mit einem herzlichen Dank für seine Unterstützung ist dieser Text Gunter Prüller-Jagenteufel gewidmet. 